Anxiety and radiotherapy

\title{
Anxiety at the first radiotherapy session for non-metastatic breast cancer: Key communication and communication-related predictors
}

\author{
Florence Lewis ${ }^{\mathrm{a}}$, Isabelle Merckaert ${ }^{\mathrm{a}, \mathrm{b}}$, Aurore Liénard ${ }^{\mathrm{a}, \mathrm{b}}$, Yves Libert ${ }^{\mathrm{a}, \mathrm{b}}$, Anne-Marie Etienne ${ }^{\mathrm{c}}$, \\ Christine Reynaert ${ }^{\mathrm{d}}$, Jean-Louis Slachmuylder ${ }^{\mathrm{e}}$, Pierre Scalliet ${ }^{\mathrm{f}}$, Paul Van Houtte ${ }^{\mathrm{a}, \mathrm{b}}$, Philippe Coucke ${ }^{\mathrm{g}}$, \\ Emile Salamon ${ }^{\mathrm{h}}$, Darius Razavi ${ }^{\mathrm{a}} \mathrm{b}, *$
}

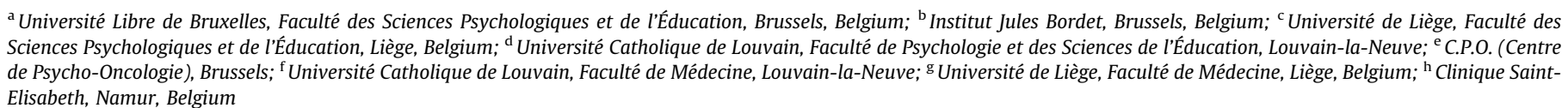

\section{A R T I C L E I N F O}

\section{Article history:}

Received 27 March 2014

Received in revised form 23 June 2014

Accepted 7 July 2014

Available online 16 September 2014

\section{Keywords:}

Cancer

Anxiety

Radiotherapy

Distress

Communication

\begin{abstract}
A B S T R A C T
Background and purpose: Patients may experience clinically relevant anxiety at their first radiotherapy (RT) sessions. To date, studies have not investigated during/around the RT simulation the key communication and communication-related predictors of this clinically relevant anxiety.

Material and methods: Breast cancer patients $(n=227)$ completed visual analog scale (VAS) assessments of anxiety before and after their first RT sessions. Clinically relevant anxiety was defined as having preand post-first RT session VAS scores $\geqslant 4 \mathrm{~cm}$. Communication during RT simulation was assessed with content analysis software (LaComm), and communication-related variables around the RT simulation were assessed with questionnaires.

Results: Clinically relevant anxiety at the first RT session was predicted by lower self-efficacy to communicate with the RT team ( $\mathrm{OR}=0.65 ; p=0.020)$, the perception of lower support received from the RT team $(\mathrm{OR}=0.70 ; p=0.020)$, lower knowledge of RT-associated side effects $(\mathrm{OR}=0.95 ; p=0.057)$, and higher use of emotion-focused coping ( $\mathrm{OR}=1.09 ; p=0.013$ ).

Conclusions: This study provides RT team members with information about potential communication strategies, which may be used to reduce patient anxiety at the first RT session.
\end{abstract}

(C) 2014 Elsevier Ireland Ltd. All rights reserved. Radiotherapy and Oncology 114 (2014) 35-41
Many studies have shown that patients undergoing radiotherapy often experience anxiety [1-7]. Anxiety during radiotherapy is associated with numerous factors, including fear of the unknown aspects of treatment [8] and side effects [7]. Previous results of this study conducted in the same sample of patients with non-metastatic breast cancer showed that patient anxiety was highest at the first radiotherapy session and that $16 \%$ of patients presented with clinically relevant anxiety. Results also underlined the need to assess patient anxiety at the radiotherapy simulation and identify patients with clinically relevant anxiety that may require additional support throughout radiotherapy [9].

Knowledge of communication and communication-related variables that affect clinically relevant anxiety may allow radiotherapy teams to improve communication during and around the radiotherapy simulation to reduce patient anxiety at the first

* Corresponding author at: Université Libre de Bruxelles, Av. F. Roosevelt, 50 - CP 191, B-1050 Bruxelles, Belgium.

E-mail address: drazavi@ulb.ac.be (D. Razavi). radiotherapy session and throughout treatment. To our knowledge, no study has prospectively assessed, during and around radiotherapy simulation, communication and communication-related predictors of clinically relevant anxiety at the first radiotherapy session.

The main objective of this study was to investigate key communication and communication-related predictors assessed during and around the radiotherapy simulation that may be associated with clinically relevant anxiety at the first radiotherapy session in patients with non-metastatic breast cancer. First, it was hypothesized that clinically relevant anxiety at the first radiotherapy session would be predicted by communication strategies utilized by the radiotherapy team (levels of assessment, information, and support skills) during the radiotherapy simulation. Second, it was hypothesized that clinically relevant anxiety at the first radiotherapy session would be predicted by lower self-efficacy to communicate with the radiotherapy team, a higher desire for support from the radiotherapy team, the perception of lower support received from the radiotherapy team, lower knowledge of side effects, 
and, consistent with the literature, lower use of problem-focused and support-seeking coping strategies [10-12].

The purpose of this study was to provide radiotherapy teams information about potential communication strategies that, if implemented during and around the radiotherapy simulation, may reduce clinically relevant anxiety at the first radiotherapy session.

\section{Material and methods}

\section{Subjects}

This study was part of a larger inter-university research program to test the efficacy of a communication skills training program conducted in four radiotherapy units in Belgium [13]. Local ethics committees approved the study. To be included in this study, radiotherapy team members had to speak French and willingly participate in the training program and assessment procedures. Radiotherapy team members were also asked to provide permission for their patients' inclusion in the study. Female patients $\geqslant 18$ years old with surgically-treated primary breast cancer without metastases who were receiving radiotherapy for the first time, had no cognitive dysfunction, and provided written informed consent were included in the study.

\section{Study design and assessment procedure}

Independent investigators recruited patients for this multicenter descriptive longitudinal study and helped them complete questionnaires. Assessments happened at the radiotherapy simulation, the first five sessions and the last five sessions of radiotherapy. For this study, only assessments at the radiotherapy simulation and the first radiotherapy session were analyzed.

\section{Questionnaires}

\section{Sociodemographic data}

Patients provided demographic information including age, marital status, educational level, occupational status, and cultural origin on a questionnaire.

Disease-related characteristics

Physicians provided data about patients' disease and treatment characteristics, including diagnoses (months since diagnosis, disease stage) and received or scheduled treatments (surgery, chemotherapy, radiotherapy, hormonotherapy, biological therapy).

Immediately before or after the radiotherapy simulation, patients completed:

The Patients' Self-Efficacy to Communicate with the Radiotherapy Team Members Questionnaire. This 5-item self-report questionnaire, developed for this study, assessed before the radiotherapy simulation patients' perceptions of their abilities to communicate with radiotherapy team members about their disease- and treatmentrelated and other concerns. Answers to questions were on a fivepoint Likert scale ranging from "not at all able" (1) to "extremely able" (5). The total score represented the mean score for all 5 items.

The Ways of Coping Checklist (WCC) [14], assessed after the radiotherapy simulation, was a 27 -item four-point self-reported measure of coping responses consisting of three subscales representing the problem-focused, emotion-focused, and social-seeking types of coping.

The Patients' Desire for Radiotherapy Team Support Questionnaire, a 9-item self-report questionnaire developed for this study, was used to assess before the radiotherapy simulation patient desire for support from the radiotherapy team. It measured three types of social support (practical, informational, and emotional) from physicians, nurses/technologists, and secretaries. This assessment also utilized the five-point Likert scale ranging from "not at all" (1) to "entirely" (5). The practical, informational, and emotional sub-scores corresponded to the mean desire for support from physicians, nurses/technologists, and secretaries. The total score represented the mean score for all 9 items.

Patients also completed just before the first radiotherapy session:

The Patients' Perception of Received Support from the Radiotherapy Team Questionnaire, a 9-item self-report questionnaire, developed for this study, was used to assess patients' perceptions of support received from the radiotherapy team during radiotherapy simulation. It measured three types of social support (practical, informational, and emotional) provided by physicians, nurses/ technologists, and secretaries. Answers were provided on a five-point Likert scale ranging from "not at all" (1) to "entirely" (5). The practical, informational, and emotional sub-scores corresponded to the mean support provided by physicians, nurses/technologists, and secretaries. The total score represented the mean score for all 9 items.

The Patients' Knowledge of Radiotherapy Side Effects Questionnaire was specifically developed for this study to assess symptoms reported as potential side effects of radiotherapy by patients. Among the 30 reported symptoms, 15 were known side effects of radiotherapy and 15 were not directly associated to radiotherapy. For each of the 30 symptoms, patients were asked if they attributed it to radiotherapy (yes or no). The "correct knowledge", a score out of 30 , was measured by adding the number of radiotherapy related side effects rightly attributed by patients to radiotherapy and the number of radiotherapy unrelated side effects rightly attributed by patients to other causes. The "incorrect knowledge", scored out of 30 , was measured by adding the number of radiotherapy related side effects wrongly attributed by patients to other causes and the number of radiotherapy unrelated side effects wrongly attributed by patients to radiotherapy. The total score (range $-30,+30$ ) was calculated by subtracting the "incorrect knowledge" score from the "correct knowledge" score.

To assess anxiety, patients were asked to report their anxiety levels using a visual analog scale (VAS) before and after the radiotherapy simulation, the first five radiotherapy sessions (first week of radiotherapy), and the last five radiotherapy sessions (last week of radiotherapy). In this study, only the VAS assessments recorded before and after the first radiotherapy session were used. The VAS consisted of a horizontal $10-\mathrm{cm}$ line, with the extreme left defined as "not at all anxious" and the extreme right defined as "extremely anxious." The VAS was used because it has been shown to be appropriate and adequate for the assessment of state anxiety [15-17], and they are easy for patients to complete. As recommended by Mitchell [18-19], we considered patients with pre- and post-first session VAS scores $\geqslant 4 \mathrm{~cm}$ to have clinically relevant anxiety and patients with pre-and/or post-first session VAS scores $<4 \mathrm{~cm}$ to have no clinically relevant anxiety.

\section{Radiotherapy team communication content analysis}

Encounters between radiotherapy team members and patients during radiotherapy simulations were audiotaped and then transcribed. Transcripts were analyzed using LaComm, French communication content analysis software. This software uses a word count strategy based on categories of words similar to PROTocol ANalyzer [20], Linguistic Inquiry and Word Count [21], and General Inquirer [22] methods. The software analyzed verbal communication utterance by utterance identifying utterance types and contents. This software is used generally in medicine, particularly in oncology.

The results discussed in the present article were based only on team members' utterances. Regarding utterances types, the communication used during the radiotherapy simulations was analyzed with LaComm dictionaries which were composed of words, word stems, or expressions built on empirical knowledge derived from actual and simulated patient consultations performed by physicians 
[23-24]. The organization of the dictionary was adapted from the Cancer Research Campaign Workshop Evaluation Manual [23-26] and was redefined and categorized according to the three-function approach of medical consultation [27] by a panel of experts. Thus, utterances were categorized in three main types: assessment, support, and information [28]. For utterance contents, four dictionaries were used: medical, radiotherapy, emotional, and social. Results of the utterance types and contents are presented in frequencies.

\section{Statistical analyses}

Descriptive data of patients with clinically relevant anxiety and without clinically relevant anxiety were collected. Associations between each potential predictor and patient anxiety were tested using parametric tests and non-parametric tests as appropriate (Student's $t$-test, $\chi^{2}$ test, and Mann-Whitney test). All tests were two-tailed, and the alpha level was set to 0.05. A backward stepwise (conditional) regression model was computed using logistic regression to assess relationships between each potential predictor and patient anxiety. All variables (total scores if available) with univariate $p$-value $\leqslant 0.10$ were entered in the model. Analyses were performed with IBM SPSS software (version 20.0; SPSS Inc., Chicago, IL, USA). Data are reported as mean \pm standard deviation (SD).

\section{Results}

\section{Subjects}

Among 340 patients approached, 47 (14\%) did not meet inclusion criteria and 47/293 (16\%) eligible patients refused to participate in the study. During the course of the study, 10 (3\%) patients stopped completing questionnaires. Only patients who completed the state anxiety VAS assessment at the first radiotherapy session were included. Four patients were excluded from the analysis because their radiotherapy simulations did not occur on the same day as simulation assessments $(n=2)$ or on the second radiotherapy simulations $(n=2)$. Five patients were excluded from the analysis because they did not receive a first radiotherapy session on the same day as the first radiotherapy session assessment. Data from 227 patients were analyzed. For the communication content analyses, 6 patients were not included due to recording problems.

\section{Patients' sociodemographic, disease, and treatment characteristics}

Table 1 displays sociodemographic, disease, and treatment characteristics of patients included in the study.

Table 1

Sociodemographic, disease, and treatment characteristics of patients ( $n=227$ ).

\begin{tabular}{|c|c|c|c|c|c|c|}
\hline & \multicolumn{5}{|c|}{ Anxiety at the first radiotherapy session } & \multirow[b]{3}{*}{$p^{c}$} \\
\hline & \multicolumn{2}{|c|}{ Clinically relevant anxiety ${ }^{\mathrm{a}}(n=41)$} & \multicolumn{3}{|c|}{ No clinically relevant anxiety ${ }^{\mathrm{b}}(n=186)$} & \\
\hline & $n$ & $\%$ & $n$ & & $\%$ & \\
\hline Age & & & & & & 0.659 \\
\hline Mean & & & & 55.7 & & \\
\hline SD & & & & 10.9 & & \\
\hline Time since diagnosis (months) ${ }^{\mathrm{e}}$ & & & & & & 0.037 \\
\hline Mean & & & & 4.5 & & \\
\hline SD & & & & 2.4 & & \\
\hline \multicolumn{7}{|l|}{ Radiotherapy ${ }^{\mathrm{f}}$} \\
\hline Number of sessions & & & & & & 0.829 \\
\hline Mean & & & & 23.3 & & \\
\hline SD & & & & 3.1 & & \\
\hline Marital status & & & & & & 0.697 \\
\hline Living alone & 12 & 29.3 & 48 & & 25.8 & \\
\hline In couple & 29 & 70.7 & 138 & & 74.2 & \\
\hline Educational level & & & & & & 0.703 \\
\hline Junior high school or less & 10 & 24.4 & 52 & & 28.0 & \\
\hline High school graduation or more & 31 & 75.6 & 134 & & 72.0 & \\
\hline Occupational status & & & & & & 0.842 \\
\hline Working part or full time & 9 & 22.0 & 47 & & 25.3 & \\
\hline Not working & 32 & 78.0 & 139 & & 74.7 & \\
\hline Stage of disease $\mathrm{f}^{\mathrm{f}}$ & & & & & & $0.236^{\mathrm{d}}$ \\
\hline In situ & 5 & 12.2 & 9 & & 4.9 & \\
\hline Stage I and II & 35 & 85.4 & 156 & & 84.8 & \\
\hline Stage III and IV & 1 & 2.4 & 19 & & 10.3 & \\
\hline \multicolumn{7}{|l|}{ Surgery ${ }^{\mathrm{g}}$} \\
\hline Lumpectomy & & & & & & 0.831 \\
\hline Yes & 32 & 78.0 & 146 & & 79.8 & \\
\hline No & 9 & 22.0 & 37 & & 20.2 & \\
\hline Mastectomy & & & & & & 0.835 \\
\hline Yes & 8 & 19.5 & 41 & & 22.4 & \\
\hline No & 33 & 80.5 & 142 & & 77.6 & \\
\hline Chemotherapy ${ }^{g}$ & & & & & & 0.230 \\
\hline Yes & 18 & 43.9 & 100 & & 54.6 & \\
\hline No & 23 & 56.1 & 83 & & 45.4 & \\
\hline Hormonotherapy ${ }^{g}$ & & & & & & 0.336 \\
\hline Yes & 27 & 65.9 & 135 & & 73.8 & \\
\hline No & 14 & 34.1 & 48 & & 26.2 & \\
\hline
\end{tabular}

a Pre- and post-session VAS score $\geqslant 4 \mathrm{~cm}$.

b Pre- and/or post-session VAS score $<4 \mathrm{~cm}$.

c Mann-Whitney $U$-test or Chi-square test.

d Goodman and Kruskal's tau.

e Missing data for 8 patients.

${ }^{f}$ Missing data for 2 patients.

g Missing data for 3 patients. 
The mean age of patients with clinically relevant anxiety at the first radiotherapy session was $56 \pm 12.1$ years (range, 27.973.6 years); $71 \%(n=29)$ were married or cohabiting, $76 \%(n=31)$ had completed high-school education, and 22\% $(n=9)$ worked part or full time. The mean time since diagnosis was $3.6 \pm 2.0$ months. The majority $(85 \%, n=35)$ of patients had stage I or II disease. Most

Table 2

Predictors of clinically relevant anxiety at the first radiotherapy session: radiotherapy team communication variables ( $n=227$ ).



a Pre- and post-session VAS score $\geqslant 4 \mathrm{~cm}$.

b Pre- and/or post-session VAS score $<4 \mathrm{~cm}$.

c Mann-Whitney $U$-test.

d Missing data for 6 patients.

e Types and contents are in frequencies.

Table 3

Predictors of clinically relevant anxiety at the first radiotherapy session: patient communication-related variables $(n=227)$.

\begin{tabular}{|c|c|c|c|c|c|}
\hline & \multicolumn{4}{|c|}{ Anxiety at the first radiotherapy session } & \multirow[b]{3}{*}{$p^{c}$} \\
\hline & \multicolumn{2}{|c|}{ Clinically relevant anxiety $(n=41)$} & \multicolumn{2}{|c|}{ No clinically relevant anxiety ${ }^{\mathrm{b}}(n=186)$} & \\
\hline & Mean & SD & Mean & SD & \\
\hline \multicolumn{6}{|l|}{ Patients' self-efficacy to communicate with the radiotherapy team } \\
\hline About their disease or treatment & 4.4 & 1.0 & 4.7 & 0.8 & 0.030 \\
\hline About their concerns related to disease or treatment & 4.0 & 1.1 & 4.5 & 1.0 & 0.003 \\
\hline About their concerns not related to disease or treatment & 3.4 & 1.4 & 3.6 & 1.6 & 0.396 \\
\hline About their anxiety related to disease or treatment & 4.1 & 1.0 & 4.4 & 1.1 & 0.006 \\
\hline About their sadness related to disease or treatment & 3.2 & 1.4 & 3.9 & 1.4 & 0.001 \\
\hline Total & 3.8 & 0.9 & 4.2 & 0.9 & 0.006 \\
\hline \multicolumn{6}{|l|}{ Patients' desire for radiotherapy team support } \\
\hline Practical support & 4.0 & 1.0 & 4.2 & 0.9 & 0.349 \\
\hline Informational support & 4.3 & 0.7 & 4.4 & 0.8 & 0.053 \\
\hline Emotional support & 3.7 & 1.1 & 3.7 & 1.1 & 0.863 \\
\hline Total & 4.0 & 0.8 & 4.1 & 0.8 & 0.436 \\
\hline \multicolumn{6}{|l|}{ Patients' perception of received support from the radiotherapy team ${ }^{\mathrm{d}}$} \\
\hline Practical support & 3.3 & 1.3 & 3.8 & 1.2 & 0.017 \\
\hline Informational support & 3.4 & 1.3 & 3.8 & 1.2 & 0.022 \\
\hline Emotional support & 2.9 & 1.2 & 3.3 & 1.3 & 0.071 \\
\hline Total & 3.2 & 1.2 & 3.6 & 1.1 & 0.018 \\
\hline \multicolumn{6}{|l|}{ Patients' knowledge of radiotherapy side effects ${ }^{\mathrm{d}}$} \\
\hline Correct knowledge & 18.7 & 3.9 & 20.0 & 3.4 & 0.059 \\
\hline Incorrect knowledge & 11.3 & 3.9 & 10.1 & 3.4 & 0.058 \\
\hline Total & 7.3 & 7.7 & 9.9 & 6.8 & 0.059 \\
\hline \multicolumn{6}{|l|}{ Patients' coping strategies (WCC) } \\
\hline Problem-focused coping & 29.6 & 6.4 & 30.7 & 5.7 & 0.330 \\
\hline Emotion-focused coping & 23.4 & 4.8 & 21.2 & 5.3 & 0.014 \\
\hline Support-seeking coping & 24.6 & 5.0 & 24.1 & 5.0 & 0.380 \\
\hline
\end{tabular}

a Pre- and post-session VAS score $\geqslant 4 \mathrm{~cm}$.

b Pre- and/or post-session VAS score $<4 \mathrm{~cm}$.

c Mann-Whitney $U$-test.

d Missing data for 1 patient.

e The Ways of Coping Checklist (WCC). 
Table 4

Predictors of clinically relevant anxiety at the first radiotherapy session: logistic regression model $(n=225)$. ${ }^{\text {a }}$

\begin{tabular}{|c|c|c|c|}
\hline & \multicolumn{3}{|c|}{ Anxiety at the first radiotherapy session } \\
\hline & OR & $95 \% \mathrm{CI}$ & $p$ \\
\hline Patients' self-efficacy to communicate with the radiotherapy team & 0.65 & 0.45 to 0.94 & 0.020 \\
\hline Patients' perception of received support from the radiotherapy team & 0.70 & 0.52 to 0.95 & 0.020 \\
\hline Patients' knowledge of radiotherapy side effects & 0.95 & 0.91 to 1.00 & 0.057 \\
\hline Patients' emotion-focused coping ${ }^{\mathrm{b}}$ & 1.09 & 1.02 to 1.17 & 0.013 \\
\hline
\end{tabular}

OR: odds ratio; 95\% CI: 95\% confidence intervals.

The goodness-of-fit of the final model had a Hosmer-Lemeshow fit statistic $\chi^{2}=5.48 ; p=0.705$.

a Missing data for 2 patients.

b The Ways of Coping Checklist.

patients (78\%, $n=32$ ) had undergone lumpectomy and approximately half $(44 \%, n=18$ ) had received chemotherapy. Radiotherapy treatment, on average, lasted $23 \pm 2.6$ sessions. Hormonotherapy was scheduled for $66 \%(n=27)$ of patients.

The mean age of patients without clinically relevant anxiety at the first radiotherapy session was $56 \pm 10.9$ years (range, 28.584.3 years $)$; $74 \%(n=138)$ were married or cohabiting, $72 \%$ $(n=134)$ completed high-school education, and $25 \% \quad(n=47)$ worked part or full time. The mean time since diagnosis was $4.5 \pm 2.4$ months. The majority of patients without clinically relevant anxiety $(85 \%, n=156)$ had stage I or II disease. Most $(80 \%$, $n=146$ ) patients had undergone lumpectomy and approximately half $(55 \%, n=100)$ received chemotherapy. Radiotherapy treatment, on average, was $23.3 \pm 3.1$ sessions. Hormonotherapy was scheduled for $74 \%$ ( $n=135)$ of patients. The two patient groups, with and without clinically relevant anxiety, did not differ in sociodemographic, disease, and treatment characteristics except for mean time since diagnosis $(p=0.037)$.

Tables 2 and 3 display radiotherapy team communication variables and patient communication-related variables predicting clinically relevant anxiety at the first radiotherapy session, respectively.

\section{Predictors of clinically relevant anxiety at the first radiotherapy} session

Regression analyses (Table 4) revealed that clinically relevant anxiety at the first radiotherapy session was predicted by lower self-efficacy to communicate with the radiotherapy team [odds ratio $[\mathrm{OR}]=0.651 ; p=0.020]$, the perception of lower support received from the radiotherapy team $(\mathrm{OR}=0.700 ; p=0.020)$, lower knowledge of radiotherapy side effects $(\mathrm{OR}=0.954 ; p=0.057)$, and higher use of emotion-focused coping $(\mathrm{OR}=1.094 ; p=0.013)$. The regression model correctly predicted $12.2 \%$ of clinically relevant anxiety cases, $97.8 \%$ of non-clinically relevant anxiety cases, and $82.2 \%$ of all cases.

\section{Discussion}

The objective of this study was to investigate key communication and communication-related variables assessed during and around the radiotherapy simulation that may predict clinically relevant patient anxiety at the first radiotherapy session. The results of this study showed that, contrary to our hypotheses, the types of communication strategies (assessment, informative, and supportive skills) used by the radiotherapy team at the radiotherapy simulation were not associated with clinically relevant anxiety at the first radiotherapy session. These results may be explained by the fact that the radiotherapy team did not engage in personalized communication with patients regarding their concerns. Clinically relevant anxiety at the first radiotherapy session was predicted by four patient communication-related variables: lower self-effi- cacy to communicate with the radiotherapy team, perception of lower support received from the radiotherapy team during the radiotherapy simulation, lower knowledge of radiotherapy side effects, and higher use of emotion-focused coping.

First, lower patient self-efficacy to communicate with the radiotherapy team at the radiotherapy simulation was, as expected, predictive of clinically relevant anxiety at the first radiotherapy session. These results indicate that patients may benefit from learning how to communicate with radiotherapy team members about their disease and treatment. Patients may also benefit from proactive assessment of disease- and treatment-related concerns.

Second, contrary to our hypotheses, clinically relevant anxiety at the first radiotherapy session was not predicted by higher desire for support from the radiotherapy team, but it was predicted by the perception of lower support received from the radiotherapy team during the radiotherapy simulation. The lack of association between clinically relevant anxiety at the first session, and desire for a higher support from the radiotherapy team may be explained by the fact that the desire for support was very high for all patients. The perception of lower support during radiotherapy simulation predicted clinically relevant anxiety at the first radiotherapy session. This result shows patients' unmet support need and indicates that patients may benefit from proactive support from the radiotherapy team addressing practical, informational, and most importantly emotional concerns.

Third, results also showed that lower knowledge of radiotherapy side effects, assessed after the radiotherapy simulation, predicted clinically relevant anxiety at the first radiotherapy session. It is important to note that both correct and incorrect knowledge predict clinically relevant anxiety at the first radiotherapy session. These results suggest that patients may benefit from proactive assessment of their knowledge of radiotherapy side effects.

Fourth, contrary to our hypotheses, clinically relevant anxiety was not predicted by lower use of problem-focused and supportseeking coping. Clinically relevant anxiety, in this study, was not predicted by patient coping strategies related to communication (e.g. information seeking, social support seeking, support seeking from professionals). Meanwhile, clinically relevant anxiety was predicted by higher use of emotion-focused coping strategies. This result is consistent with previous studies suggesting that anxiety is often associated with higher use of emotion-focused coping [1012] and indicate that patients may benefit from proactive assessment of their coping strategies. For example, the radiotherapy team could ask patients, "How do you cope with disease?" These data could help detect patients that use emotion-focused coping (e.g. avoidance, wishful thinking) and may be likely to develop emotional distress.

Fifth, it should be underlined that the variables collected during and around the radiotherapy simulation correctly predicted $12 \%$ of clinically relevant anxiety cases at the first radiotherapy session. This model gives important information with only communication and communication-related predictors. Of course, future studies should investigate other factors that may be associated with 
anxiety, such as patients' personality, life events, intolerance of uncertainty and specific treatment-related fears. Moreover, other factors directly related to the first radiotherapy session may also be associated with anxiety, such as communication strategies used by the radiotherapy team at this first session or events that would have occurred during the session (technical problems, session disruption, and discomfort in patients due to movement restriction or fear of enclosed spaces...). It should also be noted that, as it has been shown here, anxiety may also be associated with the time passed since diagnosis.

This study has several strengths. First, to our knowledge, this is the first study to prospectively investigate communication and communication-related predictors of clinically relevant anxiety at the first radiotherapy session in patients with non-metastatic breast cancer. Second, this study has specifically focused on predictors that may provide information about communication strategies, which may be used to reduce clinically relevant anxiety at the first radiotherapy session.

Previous results of this study conducted in the same sample of patients with non-metastatic breast cancer underlined the need to assess patient anxiety at the radiotherapy simulation, to identify patients with clinically relevant anxiety [9]. The present results indicate that patients may benefit from personalized communication during and around the radiotherapy simulation to prevent further unnecessary concerns. In summary, four key communication strategies should be considered. First, team members should proactively assess patients' concerns about their disease and treatment course. Second, team members should check patients' understanding and knowledge of potential side effects. After assessment, team members should provide detailed information about radiotherapy treatment and side effects to increase patients' knowledge. Third, team members should proactively assess patients' coping strategies, and note patients that employ emotion-focused coping. These patients may be at-risk for emotional distress. Fourth, team members should provide appropriate support to patients to prevent clinically relevant anxiety.

To implement personalized communication during and around the radiotherapy simulation, a systematic debriefing session should be scheduled after the radiotherapy simulation. During this debriefing session, patients may benefit from reiterating information about potential side effects. Patients should also be informed, during this debriefing session, that mental health professionals are available to help them broaden their coping resources and reduce anxiety. Of course, specific communication training programs should be available to teach radiotherapy team members to tailor their communication to patients' concerns.

The implementation of personalized communication during radiotherapy simulation may reduce patient anxiety at the first radiotherapy session. As previous results of this study have also shown, clinically relevant anxiety may of course also develop during radiotherapy treatment [9]. Team members should be aware of that and repeat the assessment of patients' anxiety at different timepoints in the course of radiotherapy treatment. Reducing patient anxiety has the potential to promote better adaptation throughout treatment. The results of this study indicate that further research is needed to assess the impacts of personalized communication on patient anxiety.

\section{Role of the funding source}

The study sponsors had no role in designing the study or collecting, analyzing, or interpreting the data. The study sponsors did not participate in the preparation, review, or approval of the manuscript.

\section{Conflict of interest statement}

The authors have no financial or personal relationships with individuals or organizations that could inappropriately influence their work. The principal investigator had full access to all study data and takes responsibility for the integrity of the data and the accuracy of the analyses.

\section{Acknowledgements}

This study was supported by the "Fonds National de la Recherche Scientifique - Télévie” of Belgium (credit number: FC 51034/ 7.4.538.07 F) and the Centre de Psycho-oncologie of Brussels. The authors would like to thank the participating hospitals and radiotherapy teams: the "Institut Jules Bordet - ULB" in Brussels (Professor Paul Van Houtte); the "Cliniques universitaires Saint-Luc" in Brussels (Professor Pierre Scalliet); the "Clinique Saint-Elisabeth" in Namur (Professor Emile Salamon), and the "Centre Hospitalier Universitaire" of Liège (Professor Philippe Coucke). The authors also thank the participating patients.

\section{References}

[1] Fritzsche K, Liptai C, Henke M. Psychosocial distress and need for psychotherapeutic treatment in cancer patients undergoing radiotherapy. Radiother Oncol 2004;72:183-9.

[2] Hopwood P, Sumo G, Mills J, Haviland J, Bliss JM. The course of anxiety and depression over 5 years of follow-up and risk factors in women with early breast cancer: results from the UK Standardisation of Radiotherapy Trials (START). Breast 2010;19:84-91.

[3] Kawase E, Karasawa K, Shimotsu S, et al. Estimation of anxiety and depression in patients with early stage breast cancer before and after radiation therapy. Breast Cancer 2012;19:147-52.

[4] Lim CC, Devi MK, Ang E. Anxiety in women with breast cancer undergoing treatment: a systematic review. Int J Evid Based Healthc 2011;9:215-35.

[5] Mose S, Budischewski KM, Rahn AN, Zander-Heinz AC, Bormeth S, Bottcher HD Influence of irradiation on therapy-associated psychological distress in breast carcinoma patients. Int J Radiat Oncol Biol Phys 2001;51:1328-35.

[6] Rahn AN, Mose S, Zander-Heinz A, et al. Influence of radiotherapy on psychological health in breast cancer patients after breast conserving surgery. Anticancer Res 1998;18:2271-3.

[7] Stiegelis HE, Ranchor AV, Sanderman R. Psychological functioning in cancer patients treated with radiotherapy. Patient Educ Couns 2004;52:131-41.

[8] Halkett GK, Kristjanson LJ, Lobb EA. 'If we get too close to your bones they'll go brittle': women's initial fears about radiotherapy for early breast cancer. Psychooncology 2008;17:877-84.

[9] Lewis F, Merckaert I, Lienard A, et al. Anxiety and its time courses during radiotherapy for non-metastatic breast cancer: a longitudinal study. Radiother Oncol 2014;111:276-80.

[10] Bolger N. Coping as a personality process: a prospective study. J Pers Soc Psychol 1990:59:525-37.

[11] Bruchon-Schweitzer M. Concepts, stress, coping. Le coping et les stratégies d'ajustement face au stress. Recherche en soins infirmiers 2001; 67.

[12] Terry DJ. Determinants of coping: the role of stable and situational factors. J Pers Soc Psychol 1994;66:895-910.

[13] Razavi D, Coucke P, Scalliet P, et al. L'optimalisation de la réponse aux besoins psychologiques des patientes traitées pour une affection cancéreuse du sein par un programme de formation à la communication destinée aux équipes de radiothérapie: une étude randomisée. Bruxelles: Projet interuniversitaire; 2005.

[14] Vitaliano PP, Russo J, Carr JE, Maiuro RD, Becker J. The Ways of Coping Checklist: revision and psychometric properties. Multivar Behav Res 1985;20:3-26.

[15] Wewers ME, Lowe NK. A critical review of visual analogue scales in the measurement of clinical phenomena. Res Nurs Health 1990;13:227-36.

[16] Miller MD, Ferris DG. Measurement of subjective phenomena in primary care research: the Visual Analogue Scale. Fam Pract Res J 1993;13:15-24.

[17] Davey HM, Barratt AL, Butow PN, Deeks JJ. A one-item question with a Likert or Visual Analog Scale adequately measured current anxiety. J Clin Epidemiol 2007;60:356-60.

[18] Mitchell AJ, Baker-Glenn EA, Granger L, Symonds P. Can the Distress Thermometer be improved by additional mood domains? Part I. Initial validation of the Emotion Thermometers tool. Psychooncology 2010;19:125-33.

[19] Mitchell AJ, Baker-Glenn EA, Park B, Granger L, Symonds P. Can the Distress Thermometer be improved by additional mood domains? Part II. What is the optimal combination of Emotion Thermometers? Psychooncology 2010;19:134-40. 
[20] Hogenraad R, Daubies C, Bestgen Y, Une théorie et une méthode générale d'analyse textuelle assistée par ordinateur: le Système PROTAN (PROTocol ANalyser). Unpublished document ed. Louvain-la-Neuve: Université Catholique de Louvain, Psychology Departement; 1995.

[21] Pennebaker J, Booth RJ, Francis M. Linguistic inquiry and word count: LIWC2007-operator's manual. Austin (TX): LIWC.net; 2007.

[22] Stone P, Dunphy D, Smith M, Ogilvie D. The general inquirer: a computer approach to content analysis. Cambridge (MA): MIT Press; 1966.

[23] Razavi D, Merckaert I, Marchal S, et al. How to optimize physicians communication skills in cancer care: results of a randomized study assessing the usefulness of posttraining consolidation workshops. J Clin Oncol 2003;21:3141-9.

[24] Delvaux N, Merckaert I, Marchal S, et al. Physicians' communication with a cancer patient and a relative. Cancer 2005;103:2397-411.
[25] Booth K, Maguire P. Development of a rating system to assess interaction between cancer patients and health professionals. London: Report to Cancer Research Campaign; 1991.

[26] Delvaux N, Razavi D, Marchal S, Brédart A, Farvacques C, Slachmuylder JL. Effects of a 105 hours psychological training program on attitudes, communication skills and occupational stress in oncology: a randomised study. Brit J Cancer 2004;90:106-14.

[27] Cohen-Cole SA. The medical interview: the three-function approach. St. Louis: Mosby Year Book; 1991.

[28] Gibon AS, Merckaert I, Lienard A, et al. Is it possible to improve radiotherapy team members' communication skills? A randomized study assessing the efficacy of a 38-h communication skills training program. Radiother Oncol 2013;109:170-7. 